\title{
Left Ventricular Hypertrophy Is More Prominent in Patients with Primary Aldosteronism than in Patients with Other Types of Secondary Hypertension
}

\author{
Akiyo Tanabe, Mitsuhide Naruse, Kiyoko Naruse, Mitsuyasu Hase, Takanobu Yoshimoto, \\ Masami Tanaka, Toshiro Seki, Reiko Demura, and Hiroshi Demura
}

\begin{abstract}
We determined functional and morphological changes of the heart by 2-dimensional and pulse Doppler echocardiography in 20 patients with primary aldosteronism and compared the results with those in 50 healthy normotensive subjects, 12 patients with Cushing's syndrome, 9 patients with pheochromocytoma, and 47 patients with essential hypertension. All hypertensive groups had greater left ventricular mass indexes than did the normotensive group $\left(76.9 \pm 17.2 \mathrm{~g} / \mathrm{m}^{2}\right)$. Despite similar age distribution, blood pressure during antihypertensive treatment, and duration of hypertension, the primary aldosteronism group had a significantly greater left ventricular mass index $\left(152.5 \pm 42.5 \mathrm{~g} / \mathrm{m}^{2}\right)$ than did the Cushing's syndrome $\left(103.4 \pm 37.5 \mathrm{~g} / \mathrm{m}^{2}\right)$, pheochromocytoma $\left(122.4 \pm 28.5 \mathrm{~g} / \mathrm{m}^{2}\right)$, and essential hypertension $(101.4$ $\pm 32.8 \mathrm{~g} / \mathrm{m}^{2}$ ) groups. The left ventricular posterior wall thickness and interventricular septal wall thickness were significantly greater in the hypertensive groups than in the normotensive group and also significantly greater in the primary aldosteronism group than in any of the other hypertensive groups. By contrast, there were no significant differences among the four hypertensive groups in any variable of systolic or diastolic function of the heart. The results suggest that left ventricular hypertrophy is more pronounced in patients with primary aldosteronism than in patients with other forms of hypertension. It is therefore important to echocardiographically evaluate cardiac hypertrophy as a risk factor of morbidity and mortality in patients with this low renin hypertension. (Hypertens Res 1997; 20: 85-90)
\end{abstract}

Key Words: primary aldosteronism, echocardiography, left ventricular hypertrophy, left ventricular mass index

Primary aldosteronism is a common cause of secondary hypertension. Diagnostic and therapeutic procedures for primary aldosteronism are well established, and this condition has been reported to be surgically curable. Measurements of plasma renin activity provide useful information for prognosis as well as diagnosis and therapy: patients with low renin essential hypertension seem to be protected from various cardiovascular events in contrast to those with normal and high renin hypertension (1, 2 ). Since plasma renin is suppressed in primary aldosteronism, it has been believed that primary aldosteronism is also a benign form of hypertension $(3,4)$.

However, the Medical Research Council studies of the incidence of cardiovascular complications in a large number of patients with primary aldosteronism revealed an unexpectedly high frequency of car- diovascular and renal events (5). Left ventricular enlargement was found electrocardiographically (6) and echocardiographically (7) in patients with primary aldosteronism. It has also been reported that aldosterone stimulates proliferation of cardiac interstitial cells, leading to cardiac fibrosis and cardiac diastolic dysfunction $(8-10)$. Although these findings suggest the importance of early diagnosis and assessment of cardiovascular complications, cardiac function in patients with primary aldosteronism has not been studied in detail.

We therefore echocardiographically evaluated functional and morphological changes of the heart in 20 patients with primary aldosteronism and compared the results with those in healthy normotensive subjects and patients with other forms of hypertension: Cushing's syndrome, pheochromocytoma, and essential hypertension.

From the Department of Medicine, Institute of Clinical Endocrinology, Tokyo Women's Medical College, Tokyo, Japan. This work was supported in part by research grants from the Japanese Ministry of Education, Science, and Culture; the Japanese Ministry of Health and Welfare "Disorders of Adrenal Hormones" Research Committee; and the Hiroto Yoshioka Memorial Medical Research Award of Tokyo Women's Medical College, awarded to H. Demura and M. Naruse.

Address for Reprints: Akiyo Tanabe, M.D., Department of Medicine, Institute of Clinical Endocrinology, Tokyo Women's Medical College, 8-1 Kawada-cho, Shinjuku-ku, Tokyo 162, Japan.

Received July 5, 1996; accepted in revised form January 21, 1997. 
Table 1. Baseline Characteristics of the Study Groups

\begin{tabular}{lccccc}
\hline & NT & EH & PA & CS & PC \\
\hline Number of patients & 50 & 47 & 20 & 12 & 9 \\
Age (yr) & $42 \pm 13$ & $47 \pm 10$ & $42 \pm 16$ & $38 \pm 17$ & $54 \pm 11$ \\
Gender (M : F) & $22: 28$ & $23: 24$ & $17: 3$ & $0: 12$ & $2: 7$ \\
Duration of hypertension (yr) & - & $7.9 \pm 6.7$ & $6.4 \pm 4.0$ & $5.2 \pm 3.2$ & $5.2 \pm 2.3$ \\
HR (bpm) & $66.2 \pm 11.1$ & $69.5 \pm 11.6$ & $65.8 \pm 10.2$ & $73.3 \pm 16.9$ & $78.1 \pm 22.1$ \\
SBP (mmHg) & $117.8 \pm 12.7$ & $149.7 \pm 19.1 *$ & $151.7 \pm 23.1 *$ & $143.9 \pm 32.2^{*}$ & $136.7 \pm 32.6^{*}$ \\
DBP (mmHg) & $70.8 \pm 11.2$ & $84.6 \pm 14.9$ & $86.4 \pm 18.3$ & $86.7 \pm 20.4$ & $71.3 \pm 12.8$ \\
MBP (mmHg) & $86.5 \pm 10.7$ & $106.1 \pm 14.1 *$ & $108.2 \pm 18.9 *$ & $105.8 \pm 23.4 *$ & $93.1 \pm 17.5 *$ \\
PAC (ng/dl) & ND & $14.4 \pm 7.0$ & $48.6 \pm 26.4^{* *}$ & $6.7 \pm 6.9$ & $1.7 \pm 8.2$ \\
PRA (ng Ang I/ml-h) & ND & $2.1 \pm 2.5$ & $0.3 \pm 0.1 * *$ & $1.3 \pm 2.0$ & $1.8 \pm 1.2$ \\
\hline
\end{tabular}

Values are the mean \pm SD. NT, normotensives; EH, essential hypertension; PA, primary aldosteronism; CS, Cushing's syndrome; PC, pheochromocytoma; M, male; F, female; HR, heart rate; SBP, systolic blood pressure; DBP, diastolic blood pressure; MBP, mean blood pressure; PAC, plasma aldosterone concentration; PRA, plasma renin activity. ND, not determined. ${ }^{*} p<0.01$ vs. NT. ${ }^{* *} p<0.01 v s$. EH, CS, and PC.

\section{Subjects and Methods}

\section{Subjects}

A total of 88 outpatients with hypertension and 50 healthy normotensive subjects were studied. None of them had a previous history of myocardial infarction, bundle branch block, long Q-T syndrome, arrhythmias, Q or Q-S patterns suggestive of infarction on baseline electrocardiograms, or echocardiographic evidence of infiltrative or valvular heart disease. The hypertensive patient groups included 20 patients with primary aldosteronism, 12 patients with Cushing's syndrome, 9 patients with pheochromocytoma, and 47 patients with essential hypertension.

The adrenal disease underlying primary aldosteronism was aldosterone-producing adenoma in 16 patients and idiopathic hyperaldosteronism in 4 patients; that underlying Cushing's syndrome was adrenocortical adenoma in 5 patients and pituitary adenoma in 7 patients. In all of the patients with pheochromocytoma, the disease was of adrenal origin and hypertension was of sustained type. The patients with primary aldosteronism were taking a calcium channel blocker, spironolactone, or both. The patients with other forms of hypertension were taking a calcium channel blocker, or angiotensin-converting enzyme inhibitor, or both.

Any antihypertensive agents were withdrawn on the day of echocardiographic study only, to minimize risk to the patients. Blood pressure was measured at the brachial artery with a standard mercury sphygmomanometer, while the subject was in the sitting position. Three consecutive readings were averaged. As shown in Table 1, there were no significant differences in baseline characteristics, including age and heart rate, among the five groups. Although blood pressures in the four hypertensive groups were significantly higher than that in the normotensive group, there were no significant differences in mean duration of hypertension or average blood pressure among the hypertensive groups.

\section{Echocardiographic Studies}

The patients were studied in partial left decubitus position with the use of standard M-mode and twodimensional echocardiograms (SONOS 500, YHP International Co. Ltd., Tokyo, Japan). Conventional parasternal short- and long-axis views were acquired to aid M-mode studies. Measurements were performed by two independent examiners, and data were expressed as an average for at least three consecutive cardiac cycles.

The following variables were determined as markers of left ventricular systolic function: left ventricular internal diameter, both at end-systole (LVIDs) and at end-diastole (LVIDd); left ventricular posterior wall thickness (PWTd) and interventricular septal wall thickness (IVSTd) at the end of diastole, defined by the peak of $\mathrm{R}$ waves; left ventricular end-systolic volume index (LVESVI); left ventricular end-diastolic volume index (LVEDVI); stroke volume (SV); ejection fraction (EF); cardiac index (CI); and left ventricular fractional shortening (FS). CI was calculated by following formula: SV $\times$ heart rate/body surface area.

Left ventricular mass (LVM) was measured in accordance with the Penn convention, using the modified cubed formula: [(LVIDd + PWTd + IVSTd $)^{3}-$ LVIDd $\left.^{3}\right] \times 1.04-13.6 \mathrm{~g}(11)$. The left ventricular mass was corrected for body surface area to obtain the left ventricular mass index (LVMI). The echocardiographic criteria for left ventricular hypertrophy were based on a LVMI > $125 \mathrm{~g} / \mathrm{m}^{2}$, as recommended by Koren et al. (12). Since spironolactone has been shown to prevent cardiac remodeling $(13,14)$, the LVMI was compared in subgroups of patients with and without previous treatment with spironolactone for primary aldosteronism.

To identify further the pattern of left ventricular hypertrophy, relative wall thickness was calculated as posterior wall thickness $\times 2 / \mathrm{LVIDd}$. The patients with left ventricular hypertrophy were then classified into the following three groups according to the increase in LVMI and the relative wall thick- 
Table 2. Functional Variable of Echocardiography in the Normotensive Subjects and Patients with Hypertension

\begin{tabular}{llllll}
\hline & \multicolumn{1}{c}{ NT } & \multicolumn{1}{c}{ EH } & \multicolumn{1}{c}{ PA } & CS & PC \\
\hline Number of patients & 50 & 47 & 20 & 12 & 9 \\
LVIDs (mm) & $27.3 \pm 2.7$ & $27.9 \pm 3.4$ & $30.1 \pm 3.4$ & $27.2 \pm 6.3$ & $27.2 \pm 4.3$ \\
LVIDd (mm) & $46.0 \pm 3.4$ & $47.7 \pm 3.3$ & $51.4 \pm 3.5^{\dagger \dagger}$ & $44.3 \pm 8.6$ & $47.2 \pm 2.8$ \\
LVESVI (m) & $17.7 \pm 3.8$ & $18.2 \pm 5.0$ & $20.7 \pm 5.3$ & $18.5 \pm 8.5$ & $19.1 \pm 6.8$ \\
LVEDVI (ml) & $61.8 \pm 9.4$ & $63.7 \pm 12.7$ & $72.9 \pm 11.6^{\dagger \dagger}$ & $59.0 \pm 23.0$ & $68.8 \pm 13.0$ \\
SV $(\mathrm{ml})$ & $70.0 \pm 12.2$ & $76.7 \pm 14.4$ & $90.0 \pm 18.3^{\dagger}$ & $59.3 \pm 27.5$ & $75.3 \pm 14.3$ \\
EF $(\%)$ & $71.3 \pm 4.0$ & $72.8 \pm 6.5$ & $71.7 \pm 7.0$ & $68.6 \pm 7.5$ & $72.1 \pm 9.2$ \\
CI $\left(1 / \mathrm{min} / \mathrm{m}^{2}\right)$ & $2.89 \pm 0.53$ & $3.23 \pm 0.88$ & $3.47 \pm 0.56$ & $2.94 \pm 1.10$ & $3.39 \pm 0.48$ \\
FS $(\%)$ & $40.6 \pm 3.5$ & $41.5 \pm 5.9$ & $41.3 \pm 5.9$ & $38.6 \pm 6.7$ & $42.3 \pm 8.3$ \\
E/A & $1.45 \pm 0.60$ & $1.00 \pm 0.28^{*}$ & $1.00 \pm 0.40 *$ & $1.15 \pm 0.45^{*}$ & $0.98 \pm 0.34^{*}$ \\
\hline
\end{tabular}

Values are the mean \pm SD. NT, normotensives; EH, essential hypertension; PA, primary aldosteronism; CS, Cushing's syndrome; PC, pheochromocytoma; LVIDs, end-systolic left ventricular internal diameter; LVIDd, end-diastolic left ventricular internal diameter; LVESVI, left ventricular end-systolic volume index; LVEDVI, left ventricular end-diastolic volume index; SV, stroke volume; EF, ejection fraction; CI, cardiac index; FS, left ventricular fractional shortening; $E / A$, ratio of early diastolic filling rate to peak rate. ${ }^{*} p<0.01 v s$. NT. ${ }^{\dagger} p<0.05,{ }^{\dagger \dagger} p<0.01 v s$. NT. EH, CS, and PC.

ness: concentric left ventricular hypertrophy (increased LVMI with increased relative wall thickness, defined as more than 0.45 ), concentric left ventricular remodeling (increased relative wall thickness without increased LVMI), and eccentric left ventricular hypertrophy (increased LVMI without increased relative wall thickness) (15).

In addition, pulse wave Doppler echocardiography was performed to determine the ratio of early diastolic filling rate to peak rate $(E / A)$ as an index of the left ventricular diastolic function (16).

\section{Blood Samples and Assay Methods}

Blood samples were taken between 9:00 and 10:00 a.m. on the day of the echocardiographic study after the patient had fasted overnight and laid supine for $30 \mathrm{~min}$. The samples were collected into EDTAtreated tubes and centrifuged; plasma samples were kept at $-80^{\circ} \mathrm{C}$ until assay. Plasma renin activity was determined as Ang I-generating activity with the use of a commercially available radioimmunoassay kit (Dainabot RI Laboratories, Tokyo, Japan). Plasma aldosterone concentration was also measured by radioimmunoassay using a commercially available kit (Dainabot RI Laboratories, Tokyo, Japan).

\section{Statistical Analysis}

Values are given as the mean \pm SD. Statistical significance was analyzed by Student's $t$-test or MannWhitney $U$-test, as appropriate. Linear regression analysis was performed with the least-squares method. The prevalence of left ventricular hypertrophy in each group was compared by chi-square test. A $p$ value less than 0.05 was considered to indicate statistical significance.

\section{Results}

There were no significant differences among the normotensive group and the four hypertensive

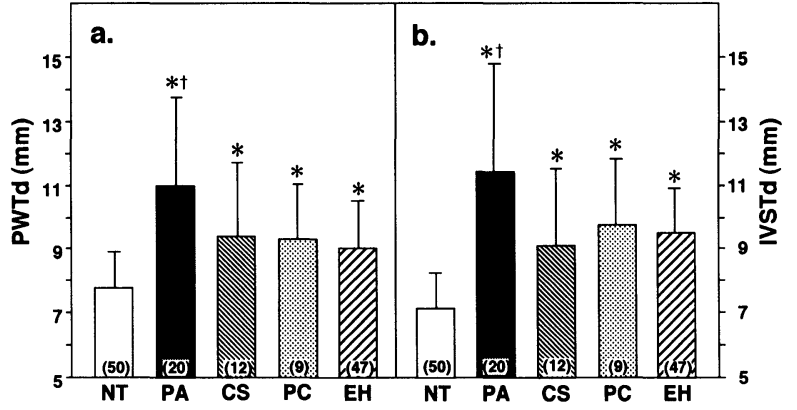

Fig. 1. Comparison of the left ventricular posterior wall thickness $(P W T d)$ and interventricular septal wall thickness (IVSTd) among normotensive subjects (NT), and patients with primary aldosteronism (PA), Cushing's syndrome $(C S)$, pheochromocytoma $(P C)$, and essential hypertension $(E H) .{ }^{*} \mathrm{p}<0.01$ vs. $N T .{ }^{\dagger} \mathrm{p}<0.01$ vs. all three other forms of hypertension.

groups in any functional variable, including LVIDs, LVESVI, EF, CI, and FS (Table 2). On the other hand, LVIDd, LVEDVI, and SV were significantly larger in patients with primary aldosteronism than in the normotensive group and patients with Cushing's syndrome, pheochromocytoma, or essential hypertension. $E / A$ was significantly lower in the hypertensive groups than in the normotensive group (Table 2).

The echocardiographic parameters of structural changes are shown in Figs. 1 and 2. PWTd, IVSTd and LVMI in all hypertensive groups were significantly greater than those in the normotensive group. Both PWTd $(11.0 \pm 2.1 \mathrm{~mm})$ and IVSTd $(11.4 \pm 2.7 \mathrm{~mm})$ in patients with primary aldosteronism were significantly larger than those in patients with Cushing's syndrome (PWTd, 9.4 $\pm 2.3 \mathrm{~mm}$; IVSTd, 9.1 $\pm 1.8 \mathrm{~mm}$ ), pheochromocytoma (PWTd, $9.3 \pm 1.2 \mathrm{~mm}$; IVSTd, $9.8 \pm 1.5 \mathrm{~mm})$, essential hypertension (PWTd, 9.0 $1.5 \mathrm{~mm}$; IVSTd, $9.5 \pm$ 
Table 3. Classification of Left Ventricular Hypertrophy according to Left Ventricular Geometric Pattern

\begin{tabular}{|c|c|c|c|c|}
\hline \multirow{2}{*}{ Ventricular geometry } & $\mathrm{EH}$ & $\mathrm{PA}$ & $\mathrm{CS}$ & $\mathrm{PC}$ \\
\hline & $n(\%)$ & $n(\%)$ & $n(\%)$ & $n(\%)$ \\
\hline Normal & $33(70.2)$ & $6(30)$ & $7(58.3)$ & $5(55.6)$ \\
\hline Concentric remodeling & $6(12.8)$ & $0(0)$ & $2(16.7)$ & $0(0)$ \\
\hline Concentric hypertrophy & $4(8.5)$ & $10(50)$ & $1(8.3)$ & $2(22.2)$ \\
\hline Eccentric hypertrophy & $4(8.5)$ & $4(20)$ & $2(16.7)$ & $2(22.2)$ \\
\hline
\end{tabular}

Values are the mean \pm SD. $n$, number of patients. EH, essential hypertension; PA, primary aldosteronism; CS, Cushing's syndrome; PC, pheochromocytoma.

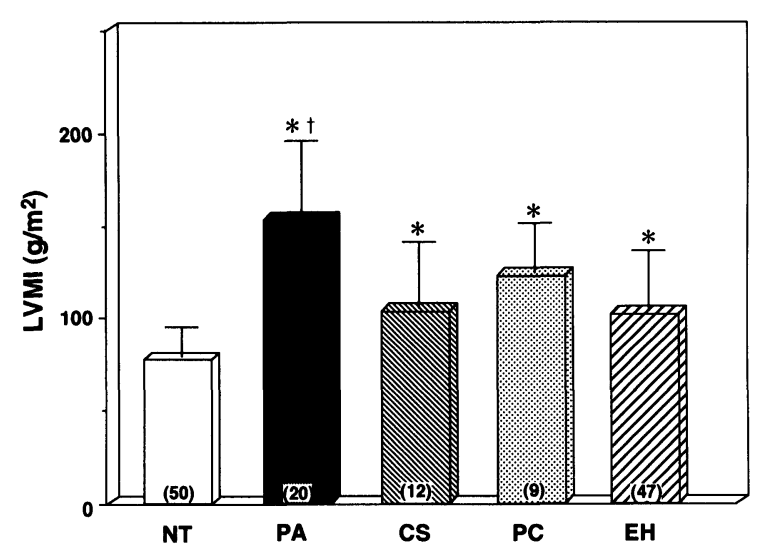

Fig. 2. Comparison of the left ventricular mass index (LVMI) among normotensive subjects (NT) and patients with primary aldosteronism $(P A)$, Cushing's syndrome $(C S)$, pheochromocytoma $(P C)$, and essential hypertension $(E H) .{ }^{*} \mathrm{p}<0.01$ vs. $N T .{ }^{\dagger} \mathrm{p}<0.01$ vs. all three other forms of hypertension.

$1.3 \mathrm{~mm}$ ), and normotensive subjects (PWTd: $7.3 \pm$ $1.1 \mathrm{~mm}$; IVSTd: $7.7 \pm 1.2 \mathrm{~mm}$ ) (Fig. 1). In addition, the LVMI in patients with primary aldosteronism $\left(152.5 \pm 42.5 \mathrm{~g} / \mathrm{m}^{2}\right)$ was markedly greater than that in patients with Cushing's syndrome (103.4 \pm 37.5 $\left.\mathrm{g} / \mathrm{m}^{2}\right)$, pheochromocytoma $\left(122.4 \pm 28.5 \mathrm{~g} / \mathrm{m}^{2}\right)$, essential hypertension $\left(101.4 \pm 32.8 \mathrm{~g} / \mathrm{m}^{2}\right)$, and normotensive subjects (76.9 $\left.\pm 17.2 \mathrm{~g} / \mathrm{m}^{2}\right)$ (Fig. 2).

The prevalence of left ventricular hypertrophy according to echocardiographic criteria (9) was $70.0 \%$ in patients with primary aldosteronism, $25.0 \%$ in Cushing's syndrome, $44.4 \%$ in pheochromocytoma, and $17.0 \%$ in essential hypertension (Table 3). In addition, the prevalence of concentric hypertrophy was $50.0 \%$ in patients with primary aldosteronism, $8.3 \%$ in Cushing's syndrome, $22.2 \%$ in pheochromocytoma, and $8.5 \%$ in essential hypertension (Table 3). The prevalence of both left ventricular hypertrophy and concentric hypertrophy was highest in patients with primary aldosteronism $(p<0.025)$.

The LVMI was compared between subgroups of patients with primary aldosteronism according to whether or not they had previously received spironolactone. The LVMI $\left(159.5 \pm 42.6 \mathrm{~g} / \mathrm{m}^{2}\right)$ in the 7 patients who had received spironolactone for 1 to 6

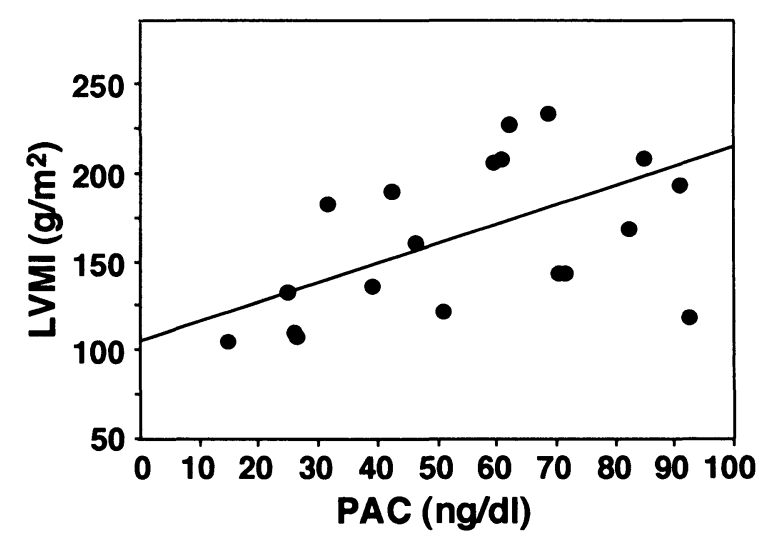

Fig. 3. Relationship between left ventricular mass index (LVMI) and plasma aldosterone concentration (PAC) in patients with primary aldosteronism.

months was not significantly different from that in the 13 patients who had not received spironolactone $\left(148.5 \pm 30.8 \mathrm{~g} / \mathrm{m}^{2}\right)$.

Although plasma renin activity was suppressed and plasma aldosterone concentration was significantly elevated in patients with primary aldosteronism, these variables were within normal limits in the other three hypertensive groups (Table 2). In the patients with primary aldosteronism, there was a slight positive correlation between LVMI and plasma aldosterone concentration $(r=0.56, p<0.05)$ (Fig. 3), but there was no correlation between LVMI and duration of hypertension, blood pressure level, or plasma renin activity (data not shown). In the patients with essential hypertension, LVMI was not correlated with the duration of hypertension, blood pressure level, plasma renin activity, or plasma aldosterone concentration (data not shown).

\section{Discussion}

In addition to stroke, cardiovascular events such as acute myocardial infarction, congestive heart failure, and sudden cardiac death occur frequently in patients with untreated essential hypertension (17). In contrast, endocrine hypertension caused by adrenal tumors is considered surgically curable and to carry a good prognosis, especially in patients with primary aldosteronism. In the present study, 
however, we clearly demonstrated by echocardiography that the LVMI, PWTd, and IVSTd are significantly increased in patients with primary aldosteronism. In addition, we found that the prevalence of concentric left ventricular hypertrophy was high in patients with primary aldosteronism. Although the LVMI in patients with pheochromocytoma also exceeded the echocardiographic criteria for left ventricular hypertrophy (12), there was no statistically significant difference from age distribution- or blood pressure-matched groups with other forms of hypertension. The significant left ventricular hypertrophy seen in patients with primary aldosteronism, therefore, appears to be a notable feature of this type of endocrine hypertension.

The degree of hypertension was rather moderate in all four hypertensive groups, probably because of prior antihypertensive treatment. In addition, $E / A$ as an index of left ventricular diastolic function did not show any significant difference among the groups despite the significant difference in left ventricular mass. We cannot completely exclude the possibility that antihypertensive treatment may have affected the cardiac structure as well as function. However, our findings are basically in agreement with those obtained in a previous echocardiographic study of 8 patients (7) and in an electrocardiographic study of 34 patients (6). Therefore, greater left ventricular mass and a higher prevalence of left ventricular hypertrophy in primary aldosteronism than in other forms of hypertension are potentially important factors, although the significance of this finding may be limited because of possible modification by antihypertensive agents.

The mechanism(s) responsible for the left ventricular hypertrophy in patients with primary aldosteronism is not known. Cardiac hypertrophy can develop in response to the increased afterload associated with systemic hypertension. Cardiac remodeling, such as hypertrophy of cardiac myocytes with interstitial and perivascular fibrosis, is a common manifestation of hypertensive cardiopathy (18). However, the right ventricle, which is not exposed to the hemodynamic effects of increased afterload, showed similar interstitial fibrosis as the left ventricle (8). Left ventricular mass has been shown to be directly related to the risk of subsequent hypertension even in normotensive adults (19). Therefore, various humoral factors in addition to blood pressure afterload have been implicated in the pathogenesis of cardiac hypertrophy $(20,21)$. In support of this hypothesis, the duration of hypertension and the blood pressure during antihypertensive treatment in the patients with primary aldosteronism were similar to those in the other hypertensive groups in our study.

Available evidence supports an important role of the renin-angiotensin-aldosterone system as a risk factor in cardiovascular events (2). Receptors for Ang II and aldosterone (22) are ubiquitously distributed on cardiac myocytes. Reactive fibrosis of the myocardium was demonstrated in an experimental model of hypertensive heart disease produced by unilateral renal ischemia, in which plasma Ang II and aldosterone levels are elevated (23). Angiotensin-converting enzyme inhibitors (24) and aldosterone receptor antagonists such as spironolactone have been shown to prevent this remodeling $(14$, 16). Since plasma renin was suppressed and plasma aldosterone was significantly increased in patients with primary aldosteronism, not renin/Ang II but aldosterone may have a key role in the development of the cardiac hypertrophy in this disease state. The positive correlation between plasma aldosterone level and LVMI shown in patients with primary aldosteronism and the stimulatory effect of aldosterone on the proliferation of the cardiac interstitial cells $(8-10)$ support this hypothesis.

In the present study, however, no correlation between plasma aldosterone level and LVMI was seen in patients with essential hypertension who had normal plasma aldosterone levels. The findings contrast with a previous study (20) showing a positive correlation between plasma aldosterone level and LVMI in untreated patients with essential hypertension. The difference can be attributed, at least in part, to the previous administration of antihypertensive agents in patients with essential hypertension in our study.

Recent studies have shown that the left ventricular hypertrophy caused by cardiac remodeling is definitely a powerful predictor (25) as well as an independent risk factor for serious cardiovascular sequelae $(10,12,25-27)$. In addition, Koren et al. (12) reported that mortality rates were higher in patients with concentric left ventricular hypertrophy than in patients with concentric left ventricular remodeling or eccentric left ventricular hypertrophy. Our present findings of significant left ventricular hypertrophy and a high prevalence of concentric left ventricular hypertrophy in patients with primary aldosteronism appear to be closely related to the unexpectedly high incidence of cardiovascular complications in this patient subgroup (5) and may contribute to increased mortality. In contrast to these cardiac structural changes, there were no significant differences in either systolic or diastolic function among the four groups with hypertension. The reason for lack of a significant difference remains unknown, but it may be related to the similar background characteristics of the patients in terms of age distribution, degree of hypertension, and treatment with antihypertensive agents.

Irrespective of the mechanism involved, because left ventricular mass is a stronger predictor of morbidity and mortality than is either blood pressure or any other conventional risk factor (27), it is important to diagnosis primary aldosteronism early and to evaluate cardiac structural changes by echocardiography. In addition, although Pringle et al. (6) showed that electrocardiographic improvement in left ventricular hypertrophy is independent of the type of treatment, the presence of significant left ventricular hypertrophy in patients with primary aldosteronism and well controlled blood pressure suggest an inherent risk in treating such patients solely with antihypertensive agents, even in the apparent absence of cardiac dysfunction. 


\section{References}

1. Laragh JH, Baer L, Brunner HR, et al: Renin, angiotensin, and aldosterone system in pathogenesis and management of hypertensive vascular disease. Am J Med 1972; 52: 633-652.

2. Mueller FB, Laragh JH: Clinical evaluation and differential diagnosis of the individual hypertensive patient. Clin Chem 199.1; 37 (10 Pt 2): 1868-1879.

3. Laragh JH, Ulick S, Januszewicz W, et al: Aldosterone secretion and primary and malignant hypertension. J Clin Invest 1960; 39: 1091-1096.

4. Conn JW: Plasma renin activity in primary aldosteronism: importance in differential diagnosis and in research of essential hypertension. JAMA 1964; 190: 222-225.

5. Beevers DG, Brown JJ, Ferriss JB, et al: Renal abnormalities and vascular complications in primary hyperaldosteronism. Evidence on tertiary hyperaldosteronism. $Q J$ Med 1976; 179: 401-410.

6. Pringle SD, Macfarlane PW, Isles CG, et al: Regression of electrocardiographic left ventricular hypertrophy following treatment of primary hyperaldosteronism. J Hum Hypertens 1988; 2: 157-159.

7. Janota $\mathrm{T}$, Hradec $\mathrm{J}$, Kral J: Heart in adrenal diseases. Cor Vasa 1992; 34: 115-122.

8. Weber KT, Brilla CG: Pathological hypertrophy and cardiac interstitium. Circulation 1991; 83: 1849-1865.

9. Abe M, Hamada M, Matsuoka H, et al: Myocardial scintigraphic characteristics in patients with primary aldosteronism. Hypertension 1994; 23 (Suppl): I-164I-167.

10. Weber KT, Sun Y, Guarda E: Structural remodeling in hypertensive heart disease and the role of hormones. Hypertension 1994; 23: 869-877.

11. Devereux RB, Reichek N: Echocardiographic determination of left ventricular mass in man. Anatomic validation of the method. Circulation 1977; 55: 613-618.

12. Koren MJ, Devereux RB, Casale PN, et al: Relation of left ventricular mass and geometry to morbidity and mortality in uncomplicated essential hypertension. Ann Intern Med 1991; 114: 345-352.

13. Brilla CG, Matsubara LS, Weber KT: Anti-aldosterone treatment and the prevention of myocardial fibrosis in primary and secondary hyperaldosteronism. J Mol Cell Cardiol 1993; 25: 563-575.

14. Brilla CG, Matsubara LS, Weber KT: Antifibrotic effects of spironolactone in preventing myocardial fibrosis in systemic arterial hypertension. Am J Car- diol 1993; 71: 12A-16A.

15. Koren MJ, Devereux RB: Mechanism, effects, and reversal of left ventricular hypertrophy in hypertension. Curr Opin Nephrol Hypertens 1993; 28: 87-95.

16. Labovitz AJ, Pearson AC: Evaluation of left ventricular diastolic function: clinical relevance and recent Doppler echocardiographic insights. Am Heart J 1987; 114: 836-851.

17. Massie BM, Tuban JF, Szlachcic J, et al: Hypertensive heart disease: the critical role of LV hypertrophy. J Cardiovasc Pharmacol 1989; 13 (Suppl 1): 18-24.

18. Anversa P, Ricci R, Olivetti G: Quantitative structural analysis of the myocardium during physiologic growth and induced cardiac hypertrophy: a review. $J$ Am Coll Cardiol 1986; 7: 1140-1149.

19. De Simone G, Devereux RB, Roman MJ, et al: Echocardiographic LV mass and electrolyte intake predict arterial hypertension. Ann Intern Med 1991; 114: 202-209.

20. Duprez AD, Bauwens FR, De Buyzere ML, et al: Influence of arterial blood pressure and aldosterone on left ventricular hypertrophy in moderate essential hypertension. Am J Cardiol 1993; 71: 17A-20A.

21. Klug D, Robert V, Swynghedauw B: Role of mechanical and hormonal factors in cardiac remodeling and the biologic limits of myocardial adaptation. Am J Cardiol 1993; 71: 46A-54A.

22. Sun Y, Weber KT: Angiotensin II and aldosterone receptor binding in rat heart and kidney: response to chronic angiotensin II or aldosterone administration. J Lab Clin Med 1993; 122: 404-411.

23. Brilla CG, Pick R, Tan LB, et al: Remodeling of the rat right and left ventricle in experimental hypertension. Circ Res 1990; 67: 1355-1364.

24. Jalil JE, Janicki JS, Pick R, et al: Coronary vascular remodeling and myocardial fibrosis in the rat with renovascular hypertension: response to captopril. Am J Hypertens 1991; 4: 51-55.

25. Levy D, Anderson KM, Savage DD, et al: Echocardiographically detected left ventricular hypertrophy: prevalence and risk factors. Ann Intern Med 1988; 108: 7-13.

26. Levy D, Garrison RJ, Savage DD, et al: Prognostic implications of echocardiographically determined left ventricular mass in the Framingham heart study. $N$ Engl J Med 1990; 322: 1561-1566.

27. Devereux RB, Koren MJ, De Simone G, et al: Left ventricular mass as a measure of preclinical hypertensive disease. Am J Hypertens 1992; 5 (6 Pt 2): 175s181 s. 